\title{
Tratamiento endoscópico del esófago de Barrett
}

\author{
José A. González-González* \\ Facultad de Medicina y Hospital Universitario Dr. José E. González, Monterrey, N.L., México
}

Se ha considerado que se puede no detectar displasia en pacientes con esófago de Barret de un 25 a 33\% de los pacientes, por lo que se han diseñado diversos abordajes uno de ellos es el método de Tissue Cypher, cual ya esta disponible comercialmente. Este no requiere proceso especial se hace con 4 muestras del bloque de parafina, y posteriormente integra diversas variables utilizando inmunofluorescencia, se analizan nueve proteínas biomarcadoras y cambios del núcleo celular, dando un puntaje de uno a 10 para riesgo de desarrollar neoplasia, en un trabajo de la Clínica Mayo, del Dr. Prasad G. lyer, con 475 pacientes, y el sistema clasificó adecuadamente los pacientes con displasia de bajo y alto grado'.

Y otro trabajo del Dr. Frei con 155 pacientes, edad promedio 62 años, 79\% hombres, con Barrett C3 M4, usando Tissue Cypher, con un seguimiento promedio de 7 años. El Tissue Cypher clasifico bajo riesgo (< 5.5) 11 pacientes (71\%) y 8 progresaron a los 5 años. Riesgo intermedio (5.5-6.4) $15 \%$ y alto (> 6.4) $47 \%$. Este método identificó a 17 de los 25 pacientes que progresaron, sensibilidad $68 \%$ y de manera correcta bajo el grado de displasia en 102 de 130 pacientes, especificidad de $78.5 \%$. siendo muy relevante ya que los pacientes sin displasia no tienen riesgo alto de desarrollar neoplasia ${ }^{2}$.

Un estudio acerca de detectar aneuploidía en Barrett por citología presentado por el Dr. Christopher Douville, evaluó 346 pacientes. Utiliza un primer usando mínima cantidad de DNA, siendo más fácil de hacer que el usar todo el genoma celular, el resultado lo analiza con deep learning y hace una clasificación que denomina Global Aneuploidy Score (GAS). Encontraron que el $50 \%$ de los pacientes con displasia de bajo grado tiene aneuploidía y alto grado el $70 \%$ y cerca del $100 \%$ de los pacientes con cáncer. Siendo importante la información, pues no todos los pacientes clasificados como de bajo grado tiene un riesgo bajo o moderado para carcinoma ${ }^{3}$. Existen otros biomarcadores que se han estudiado como el BarreGen, WATS 3D y el utilizar

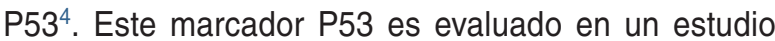
para predecir progresión de la displasia por el Dr. Mathew Stachler, trabajo retrospectivo y prospectivo, encontró que los pacientes con anormalidades de P53 sin displasia progresaron en el $49 \%$, los pacientes con displasia indefinida el $90 \%$ y los de bajo grado el $94 \%$. Teniendo una sensibilidad y especificidad en pacientes con displasia de bajo, intermedio y alto grado del 49 y $98,90 \%$ y 84,94 y $54 \%$ respectivamente ${ }^{5}$.

Como recientemente vemos en el día a día de las publicaciones, se presenta un trabajo de la Clínica Mayo utilizando el método de inteligencia artificial de enseñanza profunda en el diagnóstico histológico de la displasia en esófago de Barrett. Incluyen a 542 pacientes, 400 para entrenamiento de la prueba, 49 para validación y 48 para la prueba en sí misma. Edad promedio 62 años. El sistema reporto una sensibilidad y especificidad del 81 y $92 \%$ con un VPP y VPN del 85 y $96 \%$ para displasia de bajo y alto grado ${ }^{6}$.

El Dr. Rubén Gabriels de la Universidad Medical Center en Groninga, utiliza fluorecencia molecular más Bevamuzimab-800CW para detectar displasia en 
pacientes con Barrett, demuestra su utilidad de manera preliminar en la detección de focos de displasia, cuales prácticamente no son identificables con endoscopia de alta definición ${ }^{7}$. Otro estudio interesante es acerca del desarrollo de Barrett en los pacientes con manga gástrica de la Universidad de Colorado y realizan un análisis de incidencia y se reporta en 5,562 pacientes con manga gástrica (2017-19) una baja incidencia de esófago de Barrett a 5 años $(6.4 \%)^{8}$.

En relación con la recomendación actual de los diversos tratamientos endoscópicos se sugiere revisar las guías Europeas de Tratamiento de Esófago de Barrett, publicadas $2017^{9}$.

Un estudio de la Universidad de Cambridge, acerca del uso de microscopia con focal láser en combinación con marcadores biomoleculares para el diagnóstico de displasia y reportan que este método disminuye el número de biopsias para y su uso aun en médicos sin experiencia con este método resulto satisfactorio ${ }^{10}$.

El Dr. Fasullio presenta un trabajo multicéntrico de displasia, de manera retrospectiva (2010-2019) comparan a los pacientes tratados con radiofrecuencia vs. crioterapia, el porcentaje de erradicación de la metaplasia intestinal fue similar (76 vs. $68 \% ; p=0.46$ ). La radiofrecuencia necesito menos sesiones (2.4 vs. 3.9; $p=0.01)$. Los que fallaron se cambiaron de tratamiento y la erradicación fue semejante, se sugiere que la radiofrecuencia parece ser mejor costo-efectiva ${ }^{11}$. El Dr. Kobayashi, de la Universidad de Toronto, presenta un trabajo de la erradicación completa de la metaplasia intestinal en Barrett y la recurrencia de displasia, estudio retrospectivo del 2001 al 2019, con 381 pacientes tratados con resección endoscópica o radiofrecuencia se pudieron seguir a 345 pacientes. La recurrencia en el grupo total fue de 20 pacientes (5.8\%), en 8 pacientes de 52 que no tuvieron erradicación completa (10\%), y en 12 pacientes con erradicación completa (4.5\%). Por lo que pacientes con erradicación completa tiene menor recurrencia de la displasia ${ }^{12}$.

En el Congreso Europeo 2020 se presentó un trabajo de erradicación de Barrett, donde tratan a los pacientes con argón plasma a $60 \mathrm{~W}$ después de inyección submucosa con salina vs. radiofrecuencia con un seguimiento a 6, 12 y 24 meses. Incluyen a 103 pacientes. La erradicación a 6 meses con radiofrecuencia fue del 87 vs. 91\% con APC híbrido, siendo similares en su erradicación, pero el grupo de argón plasma tuvo menos dolor y estenosis ${ }^{13}$.

Un estudio multicéntrico (inglés y estadounidense), compara la seguridad y eficacia del uso de Endorotor en la resección mucosa en pacientes con Barrett refractario a erradicación. Lo presenta el Dr. Hussein, que evalúa a 11 pacientes con Endorotor y 5 con ablación, en dos sesiones se logró la resección con Endorotor de Barrett C1 M2, sin perforación ni dolor severo, por lo que se considera seguro ${ }^{14}$.

Un estudio holandés presentado por el Dr. van Munster, del grupo de Dr. Bergman, evalúa el uso de un sistema de ablación de radiofrecuencia con agua, llamado Aqua RFVA System (Aqua Medical, Inc.). Este genera vapor de agua a 100 grado centígrados, utilizando radiofrecuencia. Se aplica por el canal de trabajo del endoscopio y no es de contacto, demostró ser seguro y es la primera prueba en humanos ${ }^{15}$. Este mismo grupo presenta un trabajo acerca del seguimiento posresección radical de adenocarcinoma de la mucosa y de la submucosa en 120 pacientes con Barrett. Estudio realizado del 2008-2019. Aquellos pacientes con invasión linfovascular y T1a de alto riesgo se asoció a muy alta probabilidad de tener presencia de neoplasia en ganglios y metástasis ${ }^{16}$.

Podemos concluir que los pacientes con displasia de alto grado deben de ser reevaluados por patólogos expertos y en centros expertos de ser posible, cabe mencionar que no existe la definición de "expertos» en estos momentos. Sabemos que un gran número de estos pacientes con displasia de bajo grado cambia su estatus postevaluación y las herramientas mencionadas como el Tissue Cypher, aneuploidía o P53 debemos considerarlas.

La estratificación de riesgo de desarrollo de esófago de Barrett se ha evaluado cada vez con más frecuencia. Podemos decir que la clasificación propuesta de manera clínica cual incorpora edad, obesidad, tabaquismo, antecedente familiar y más marcadores biomoleculares, y podrá caracterizar en un futuro mejor a los pacientes que deben de tener escrutinio para Barrett.

Los trabajos acerca de métodos no invasivos como el uso de citoesponja, EsoGuard, EsoCheck y pruebas de aliento para detectar el esófago de Barrett cada vez son más estudiados, con resultados prometedores ${ }^{17}$.

El tratamiento endoscópico se ha centrado en la erradicación por mucosectomía tanto por resección como por disección, además de métodos de radiofrecuencia, crioterapia y argón plasma como los más frecuentemente usados en la clínica ${ }^{18}$.

\section{Financiamiento}

La presente investigación no ha recibido ayudas específicas provenientes de agencias del sector público, sector comercial o entidades sin ánimo de lucro. 


\section{Conflicto de intereses}

\author{
Los autores declaran que no hay conflicto de \\ intereses.
}

\section{Bibliografía}

1. Prasad lyer, D Chamil Codipilly, Apoorova Chandar, Siddharth Agarwal et al. Prediction of Progression in Barrett Esophagus using tissue system pathology test; A pooled Analysis of international multicenter studies (lecture presentation) [Internet]. Digestive Disease Week ${ }^{\circledR} 2021$ to Showcase the TissueCypher ${ }^{\circledR}$ Barrett's Esophagus Assay as a Paradigm-shifting Precision Medicine Tool, Mayo Clinic and AMC Amsterdam to Present Data on Predicting Progression to Esophageal Cancer in Barrett's Esophagus; 17 de mayo de 2021. Disponible en: https://www.globenewswire.com/news-release/2021/05/17/2230758/0/en/Digestive-Disease-Week-2021-to-Showcase-the-TissueCypher-Barrett-s-Esophagus-Assay-as-a-Paradigm-shifting-Precision-Medicine-Tool.html

2. Frei NF. Tissue Cypher objectively risk stratifies Barrett's patients with low grade dysplasia (lecture presentation) [Internet]. Digestive Disease Week ${ }^{\circledR}$ 2021 to Showcase the TissueCypher ${ }^{\circledR}$ Barrett's Esophagus Assay as a Paradigm-shifting Precision Medicine Tool, Mayo Clinic and AMC Amsterdam to Present Data on Predicting Progression to Esophageal Cancer in Barrett's Esophagus; 17 de mayo de 2021. Disponible en: https://www. globenewswire.com/news-release/2021/05/17/2230758/0/en/Digestive-Disease-Week-2021-to-Showcase-the-TissueCypher-Barrett-s-Esophagus-Assay-as-a-Paradigm-shifting-Precision-Medicine-Tool.html

3. Christopher Douville MD. Using aneuploidy to assess progression in patients with Barret's esophagus. Presentación oral. Gastroenterology, Vol 160, Issue 6, S-12

4. ASGE Technology Committee, Trindade AJ, Navaneethan U, Aslanian HR, Bhutani MS, Krishnan K, Lichtenstein DR, et al. Advances in the diagnosis and surveillance of Barret's esophagus (with Video). Gastrointest Endosc. 2019;90(3):325-34

5. Redston M, Noffsinger A, Kim A, Akarca FG, Stapleton D, Nowden L, et al. Abnormal TP53 predicts risk of progression in patients with Barrett's esophagus regardless of a diagnosis of dysplasia [Internet]. medR $\chi$ ix 21 de octubre de 2020. Disponible en: https://www.medrxiv.org/content/10.1101/2020.10.18.20213561v1.full

6. Codipilly DC, Vogelsang D, Agarwal S, Dhaliwal L, Lansing RM, et al Utilization of a Deep learning artificial intelligence model in the histologic diagnosis of dysplasia in Barrett's esophagus. GIE AB. 2021.288;93(65).

7. Gabriëls RY, Hooghiemstra WTR, Schmidt I, van der Laan JJH, Buist-Homan M, Kats-Ugurlu G, et al. Fluorescence molecular endoscopy shows promising results in detecting dysplastic esophageal lesions using topically administered bevacizumab-800CW: The preliminary results of a phase 2 study [Internet]. DDW ${ }^{\circledR}$ 2021IVirtual, sesión 4250; 21-23 de mayo. Disponible en: https://eventpilotadmin.com/web/page.php?page = IntHtml and project = DDW21 IITE and id $=3525271$
8. Eric Swei, Shelby Sullivan, Jason Samuel, L Helmkamp, Sachin Wani, Frank I. Scott et al ID 3524509, AB $290 \mathrm{GIE} ; 90: 6 \mathrm{~S} ; 2021$

9. Weusten B, Bisschops R, Coron E, Ribeiro MD, Dumonceau JM, et al. Endoscopic management of Barrett's esophagus; European Society of Gastrointestinal Endoscopy (ESGE) Position paper. Endoscopy. 2017;49(02):191-8.

10. Vithayathi M. Confocal laser endomicroscopy in combination with molecular biomarkers for the diagnosis if inconspicuous dysplasia in Barrett's esophagus; A randomized cross-over trial. Gastroenterology. 2021;160(6):S-48.

11. Fasullo M, Patel M, Harris S, Hassouneh R, Shah T, Smallfield G. Comparasion of radiofrequency ablation to spray liquid nitrogen cryotherapy in the management of dysplasia in Barrett's esophagus: A single center experience. Gastrointest Endosc [Internet]. 2021;93(6 Suppl):AB281-AB282. Disponible en: https://www.giejournal.org/article/ S0016-5107(21)00833-6/abstract

12. Kobayashi R, Calo NC, Mosko JD, May GR, Teshima C. Complete eradication of intestinal metaplasia in patients with Barrett's esophagus with advanced neoplasia prevents recurrent dysplasia. Gastrointest Endosc [Internet]. 2021;93(6 Suppl):AB276. Disponible en: https://www. giejournal.org/article/S0016-5107(21)00823-3/pdf

13. Knabe M, Wetzka J, Kronsbein RJH, Welsh L, May A, et al. Hybrid argon plasma coagulation vs. radiofrequency ablation in Barrett's esophagus after endoscopic resection of neoplastic lesions. A randomized trial at a tertiary center. United European Gastroenterol J. 2020;8(8 Suppl):20.

14. Hussein M, Sami S, Lovat LB, Haidry R, Wang KK. Mo1280 Prospective multicenter randomized controlled trial comparing the safety and effectiveness of the Endorotor ${ }^{\circledR}$ mucosal resection device with continued ablation in the treatment of refractory Barrett's oesophagus: Report of initial outcomes. Gastrointest Endosc [Internet]. 2021;91(6 Suppl):AB414. Disponible en: https://www.giejournal.org/article/S0016-5107(20)32714-0/ fulltext

15. van Munster SN, Pow RE, Weusten BL, Bergman J. Mo1240 First in-human $(\mathrm{FIH})$ study on the safety tolerability and efficacy of the Aquamedical focal RF- Vapor ablation (RFVA) System for the eradication of Barrett's esophagus: The steam-be dosimetry study. Gastrointest Endosc [Internet]. 2020;91(6 Suppl):AB391-AB392. Disponible en: https://www. giejournal.org/article/S0016-5107(20)32674-2/fulltext

16. Van Munster S, Verheiji E, Pouw RE, Weusten BLAM, Bergman JJ. P0138 First human study on the safety tolerability and efficacy of the Aquamedical focal RF- Vapor Ablation (RFVA) system for the eradication of Barrett's esophagus (BE); The Steam-BE dosimetry study. UEG Journal. Abstract Book. 2020;8:206.

17. Poppers DM, Gross SA, Helm D. ID 3524401 EsoGuard/EsoCheck: A novel, simple oupatient technology for the early detection of esophageal intestinal metaplasia displasia and adenocarcinoma. Gastrointest Endosc [Internet]. 2021;93(6 Suppl):AB284. Disponible en: https://www. giejournal.org/article/S0016-5107(21)00840-3/fulltext

18. Manner H, May A, Kouti I, Pech O, Vieth M, Ell C. Efficacy and safety of Hybrid-APC for the ablation of Barrett's esophagus. Surg Endosc. 2016;30(4):1364-70. 